and the most gradual extension can be made. So insuperable an obstacle does this appliance offer to muscular action, that with it the advantage of reducing the muscles of the calf by bending the leg on the thigh appeared of no moment whatever. I strikingly perceived, in this instance, the trath of a remark often made by Liston, that even if a muscle be put on full stretch, it soon loses its tonicity, becomes flaccid, and ceases to act as a displacing agent. In this way, then, if you can only procure an apparatus which shall resist muscular action completely, you need not attend to position (quoad muscular action) at all; it only becomes necessary to relax the fibre when one cannot tire it out by perfect resistance.

24th.-Going on well; the leg is getting into better shape; the shaft of the tibia is much less oblique, straighter, and longer. This affords a remarkable proof of the susceptibility of callus, whilst in a soft state, to undergo stretching and moulding.

27 th.-Very much better.

Aug. 4th.- Heel has become exceedingly sore, tender, and bleeds profusely; but for this unfortunate compilication, I believe I should have succeeded in making the limb of the same length as the other, notwithstanding the removal of the end of bone. Discontinue all traction, and place the limb in the straight position merely.

14th.-Can move the entire limb easily; complete consolidation has taken place. Shortly after this, he left his bed, and took to crutches, daily gaining strength, and being possessed of a limb, which, though shorter than its fellow, is yet so useful, that he would not, in the words of Sir Astley Cooper, s5 exchange it for a wooden one for all Europe."

Suffolk Cottage, New Shoreham, Sussex, 1849.

\section{ON THE TOXICOLOGICAL PROPERTIES OF THE BRASSICA NAPUS, OR WILD CORN KALE.}

Bx JOHN POPHAM, A.B., M.B.,

PHYSICIAN TO THA CORK NORTH INFIRMARY AND UNION WOREHOUSE.

IN the months of June and July of the present year, when very great destitution prevailed in the southern and western parts of Ireland, I was surprised to find a large number of persons from some of the famine-stricken districts of the Cork union applying for admission into the workhouse hospital, with very peculiar and alarming symptoms. They were chiefly the families of country labourers out of employment, emigrating from place to place in search of work, and totally deficient of means to procure the merest necessaries of life. They all exhibited indications of deteriorated health, the hue of the skin being uniformly sallow and muddy-looking, totally different from the brown discoloration cansed by exposure to the sun; the surface was also covered with that colourless downy hair, so characteristic of what the country people here call "impoverished blood." An cedematous state prevailed generally of the whole body, but always of the face and lower extremities, the former being sometines swollen to an enormous extent, the distended state of the eyelids and upper lip especially producing remarkable deformity. The abdomen was tympanitic; the bowels torpid: the mucous membrane of the mouth and pharynx was inflamed, and in some parts ulcerated, and the gums spongy; the appetite was usually greater than natural, in some cases voracious; the urinary secretion was deficient and irritating, and that of the skin suspended; very distressing frontal headach and tension was also complained of; but the most curious symptom, next to the odematous appearance of the patient, was the state of the hands and feet, which were dry and shrunken, with blotches of a deep-red, resembling burns, on the backs of the hands and dorsum of the feet, the fingers and toes being frequently cold and livid. Similar blotches existed on the nose and forehead, and these varied in their consequences from simple discoloration to ulceration of a most troublesome description, terminating in loss of the cuticle and dropping off of the nails, with a marked disposition in the aggravated cases to gangrene. The œdematous ap pearance of these patients was so like that of persons suffering from extreme cardiac or renal disease, that $I$ formed at first a very unfavourable prognosis, but on a closer inquiry, I discovered that all the persons thus affected had made use of the field speeies of brassica as food. Some of the worst cases had used it almost as the sole means of support; others again had partaken of it at longer intervals, and with the addition of Indian meal or other farinaceous food. From the similarity of some of the symptoms to those produced in the human body by the acrid properties of the ranunculaceæ, I thought it possible that some plants of this family might by mistake have been gathered with the kale, but the identity of the symptoms in all the persons, who were from very different parts of the country, led me to abandon the idea. This was further confirmed by showing the patients the respective plants, when, in all instances, they at once distinguished the yellow racemes and cordate leaves of the Praiseach buidhe, or yellow -kale, from any other.

They stated that their mode of cooking the herb for food was to strip the leaves, rejecting the stalk, root, and flower, and to boil them with common salt or soda. Most of them had used the corn kale for a period extending from one week to six before the unfavourable effects became manifest, and these were more rapidly produced in persons previously suffering from gastric or intestinal disturbance.

I am not aware if the effects above described, and which at the time I thought it my duty to endeavour to prevent, by pointing out the injurious properties of the wild corn kale as an esculent, in the local papers, have been heretofore observed. The family of plants to which it belongs-namely, the cruciferæ, have always borne a good reputation for salutary properties; but in many of its genera there exists a volatile oily principle, peculiarly irritating to the skin and digestive organs. This volatile oil, which in some genera, as the mustard tribe, resides more particularly in the seeds, and in others in the root, exists in the wild varieties of the colewort tribe also in the leaves, which give to the tongue a disagreeable biting taste. By cultivation this acrid property becomes more diffused and diluted. The quantity of nitrogen also which the cruciferæ contains, affords great facility for the formation of ammoniacal products, and the tympanitic condition of the abdomen is most likely due to this cause. The analogy which exists between the enfeebled capillary circulation and ulcerative tendency in the extremities arising from the use of the corn kale, to the more marked gangrenous effects produced by the ergot of rye is apparent; but whether it would have proceeded to sphacelus, I am not able to say, as, fortunately, the unsightly appearance presented by the patient led them to apply early for relief. The treatment used in these cases may in all of them be successful. It consisted of such medicines as were best adapted to restore the action of the skin and kidneys, together with mild purgatives, warm baths, rest in the horizontal position, and wholesome food. The state of the extremities required careful stimulation until the circulation was restored, when the tepid water dressing was the best application. It was some time before many of these patients regained their former health, and in some the blotches have left a permanent mark.

Camden-place, Cork, 1849 .

\section{NOTES OF CASES IN PRIVATE PRACTICE.}

BY A. C. SELKIRK, M.D., Carluke.

Delirium Tremens; Chloroform.-Sometime in the beginning of February last, I was summoned to see J. H-, a young, unmarried man, who, the messenger stated, " had gone out of his wits." I found the patient labouring under a severe attack of delirium tremens. His actions and language were of the most whimsical description. Having read a good deal, the illusions, in this instance, assumed rather a scientific direction. In particular, he described to me an electric communication between his bed and the locality of his previous midnight orgies, by means of which he communicated and received the most ludicrous information imaginable.

The treatment, in the first instance, consisted of opiates alone. Large, frequently-repeated, and heroic doses, had no effect whatevor in inducing sleep, but rather produced an aggravation of the symptoms. On the second day, opium was exhibited, in combination with the wonted stimulus, with no better success. It may be stated, "Tincture of opium, twenty drops, every two hours, until the patient sleeps," was also tried, as recommended by Dr. Watson, in his "Lectures on the Practice of Physic," published in the pages of the Medical Gazette several years ago. This method I found also unavailing.

On the fourth day, from the obstinate nature of the attack, the length of time the patient had been without sleep-about a week-I determined to try chloroform. I had no precedent for the practice; and from the peculiar irritability of the nervous centre in this disease, the determination was followed out rather as a dernier ressort than as a matter of choice. A teaspoonful, of specific gravity 1.480, was placed upon a pockethandkerchief, and held to the patient's mouth. Contrary to expectation, he appeared to relish the inhalation exceedingly, when at once he exclaimed, "I'm going !" and threw his toes 
almost to the ceiling of the bed; the whole body became violently convulsed for a few seconds, requiring the united efforts of another young man and myself to keep the patient in bed; gradually the muscular system relaxed, the head dropped on the pillow, and he slept. The patient dozed for the next twelve hours almost uninterruptedly. At my visit next day, he expressed himself as being nearly quite well, and thankful for the relief obtained from the use of the chloroform. His feelings, he remarked, before its exhibition, were of the most painful and disagreeable nature; but during the whole of the night subsequent to its inhalation, the illusions were of the most agreeable description.

I have observed, in THE $\mathrm{L}_{\text {ANCET, since the above-mentioned }}$ period, several cases of delirium tremens treated with chloroform, but no mention of the phenomenon of convulsions, as remarked in the above case. It presents, therefore, some interesting points, from that circumstance alone.

Traumatic Tetanus : Chloroform.-James L_-_, miner, early in the month of February last, was subjected to amputation of the great toe and two adjoining toes of the right foot, in consequence of a piece of metal pipe falling on his foot, from the depth of several fathoms, crushing the toes to a jelly. Tho integuments left to cover the denuded bone sloughed a way, leaving by the eighth day a healthy granulating surface. On the ninth day, "slight stiffness" complained of in the motion of the jaw, trifling, indeed, but sufficient in amount to arouse suspicions of the probable accession of trismus. The wound, at this period, had assumed that morbidly florid appearance, remarked by Dr. Burns, in his "Principles of Surgery," as frequently observed previous to the appearance of tetanic symptoms.

During the three following days the rigidity of jaw increased; dyspnoa, with slight difficulty of deglutition. The patient was now brought rapidly under the specific influence of mercury. The symptoms, however, continued to increase in intensity.

On the fourteenth day: Dyspnœa urgent; extreme difficulty of deglutition; pain and feeling of "tightness" across the præcordia. Dreading the near approach of spasmodic action, $I$ resolved to give chloroform a fair trial. In a few seconds after the commencement of the inhalation, (the patient was sitting in the semi-erect position, gasping for breath, his head dropped on the pillow, the breathing became easy; the pulse fell from 115 to 95 ; and he dozed for two hours. At my next visit, six hours after the exhibition of the chloroform, I found the patient much in the same condition as before the exhibition of the agent, with this difference, that the jaw was a little more relaxed, and deglutition was effected with less difficulty. At the urgent solicitation of the patient, the anæsthetic agent was again administered, with similar results.

From this period up to the twenty-second day, chloroform was had recourse to at regular intervals, every sixth hour; slight spasmodic action several times supervened, but the chloroform instantly checked it. The patient died, apparently exhausted, on the twenty-second day. For two days previous to patient's decease, the jaw became more relaxed, and deglutition was effected with less difficulty. He never lost, however, the peculiar tetanic expression of countenance.

In the former case, (delirium tremens, the exhibition of chloroform produced convulsions. In the latter, it both acted as a prophylactic and checked the symptoms indicative of the approach of spasms. Although considerable, nay, even great benefit was derived from its use in this case, the result accorded with my expectation. If the morbid phenomena are referrible to inflammatory action of the spinal column, the shock at the moment of accident, with subsequent irritation and inflammation, the therapeutic effect of the agent must be merely palliative. Were I to meet with a similar case, I would put in practice the following method:-Place the system rapidly under the influence of mercury; counteraction along the whole course of the spine; purgatives; turpentine enemata, with the use of chloroform, and this, I am persuaded, with some chance of success. In the treatment of a disease in which the pathology is so little understood, the therapeutic arrangement must in some measure be guided by whatever theory the practitioner summoned adopts.

Dislocations.-The papers of Mr. Spong, lately published in The Lancer, I perused with no slight degree of interest, bearing, as they evidently do, the stamp of practical and original value. The simple and ingenious appliances, particularly, to aid in the reduction of dislocations, appeared ingenious, if not, also, of considerable practical interest.

Since the publication of his paper on dislocations of the shoulder-joint, I have met with a case in which the proposed method was tested satisfactorily, J, H-, a young farmer in this neighbourhood, sustained dislocation of the right shoulder-joint. I saw him eight hours after the accident. He is a powerful, muscular man, and a glance at the limb satisfied me that reduction by the heel and extension would be effected with difficulty: the trial was made, however, in the usual way, keeping up the extension steadily until my new powers were exhausted, but failed to lift the head of the bone from its seat in the axilla. Mr. Spong's method was now had recourse to, but failed for the following reason:- The towel knotted above elbow-joint, and tied behind back of operator, gives, no doubt, sufficient power of extension, but the operator's limb (heel) fails to keep up a sufficient amount of counter extension. I am satisfied that the first trial on a powerful patient will satisfy even Mr. Spong on this point. The following method which, for some time, $I$ have been in the habit of adopting, in all cases where considerable force was indispensable, succeeds almost instantly. The heel is placed in the axilla; the operator then proceeds to make extension in the usual way; desire an assistant (there is not a case in a hundred but there is some person at hand) to grasp the instep of operator's foot with one hand, and the heel with the other, and make steady but strong traction. A force is thus acquired sufficient in amount to reduce any recent dislocation of the shoulder-joint, even in the most powerful subject. In the case alluded to, this method succeeded in a few seconds. Mr. Spong's substitute for the pulley in dislocations of the hip-joint, I have not had an opportunity of testing, but should think it would answer the purpose admirably; and it carries with it the recommendation that the operation can be proceeded with instanter.

Iodide of Potassium.-Ricord, in speaking of the properties of this salt, particularly as it influences the symptoms and characteristic phenomena of tertiary accidents, remarks that large doses seldom or never cause vomiting. I am of opinion, from a considerable experience in the use of the salt, that it is one of the most powerful agents we possess of allaying the irritability of the stomach; and what seems of more practical value, the property the salt exhibits, in combination with infusion of quassia, of allaying the sympathetic irritability of that viscus, consequent on some distant local irritation-for example, in the constitutional irritation manifested in lumbar or gluteal abscesses. At the period of writing these notes, I have a case of gluteal abscess under treatment, which illustrates the practical interest of the remarks submitted. Local depletion, counteraction with usual remedies, failed to produce resolution; pus formed deep under the gluteal muscles, and was discharged by puncture. The constitutional disturbance at this period was not great, albeit the disease had existed for six months. The general irritation, however, became violent after the abscess had been discharging for six weeks, the stomach rejecting all the ingesta, both solids and fivids. Opium, small doses of castor oil, with the neutral salts, quinine, \&c., were had recourse to, without the slightest mitigation in the symptoms; the patient lost flesh rapidly. Pulse 110, weak; he was evidently fast passing into an extreme anæmic condition. At this juncture he was ordered-Iodide of potass, two drachms; infusion of quassia, two pounds. To take half a wineglassful three times daily. The vomiting and irritability of stomach ceased after the third dose, the secretions of the alimentary canal improved, and the patient, from that period, rather gained ground. He is clearly, however, of the strumous diathesis, and although the abscess has no connexion with hip.joint nor psoas muscles, still, from its extent, dissecting deep under the gluteal muscles, the great amount of constitutional disturbance set up, the prognosis is unfavourable. I have used the iodide in several cases of the above-mentioned description, with similar results. I am not aware of this property of the salt having been previously adverted to.

\section{保orégn 画epartment.}

\section{Prophylactic Syphilitic Inoculation.}

Sout time ago, when syphilis was fancied to be incompatible with cholera, a person gravely proposed to spread syphilis by inoculation, and to poison the community wholesale, in order to guard them against cholera. We perceive, now, that $M$. DIDAY, lately surgeon to theVenereal Hospital of Lyons, hasa plan pretty similar to the above, for shielding young people from the effects of the syphilitic virus. He proposes no less than to inoculate people with the venereal disease, on the same principle upon which the inoculation of small pox was grounded. M. Diday says, that constitutional syphilis attacks a man but once in his life; and from the analogy of small pox, 